\title{
Research on the Application of Improved Fuzzy-PI Control in STATCOM
}

\section{Zhao Zhenxing, Liu Fugui, Wang Yangang}

Electromagnetic Field and Electrical Reliability Laboratory, Hebei University of Technology, Tianjin, China

\section{Email address:}

1410961015@qq.com (Zhao Zhenxing),2974513115@qq.com (Liu Fugui),1260737215@qq.com (Wang Yangang)

\section{To cite this article:}

Zhao Zhenxing, Liu Fugui, Wang Yangang. Research on the Application of Improved Fuzzy-PI Control in STATCOM. Science Discovery. Vol. 4, No. 1, 2016, pp. 39-44. doi: 10.11648/j.sd.20160401.17

Received: April 2, 2016; Accepted: April 13, 2016; Published: April 18, 2016

\begin{abstract}
According to some shortcomings that Fuzzy PI Control strategy can not meet certain occasions where higher control accuracy is required, the improved Fuzzy-PI control is applied to the static synchronous compensator (STATCOM). Simulating and analyzing the compensation effects of Fuzzy-PI control strategy, improving the membership function of the Fuzzy-PI control strategy, and testing the compensation performances of the improved Fuzzy-PI control with the MATLAB/SIMULINK simulation experiments. The simulation and experimental results show that the improved Fuzzy-PI control can improve the control precision, and can meet the requirements of power system for reactive power compensation better.
\end{abstract}

Keywords: Improved Fuzzy-PI Control, Static Synchronous Compensator, Membership Function, Reactive Power Compensation

\section{改进型模糊PI控制在STATCOM中应用研究}

\section{赵振兴, 刘福贵, 王彦刚}

电磁场与电器可靠性实验室, 河北工业大学, 天津市, 中国

\section{邮箱}

1410961015@qq.com(赵振兴), 2974513115@qq.com(刘福贵), 1260737215@qq.com(王彦刚)

\begin{abstract}
摘要: 静止无功发生器 (STATCOM) 的控制策略采用的是改进型模糊PI控制策略, 仿真分析模糊PI控制策略的补偿效果, 模糊PI控制策略无法满足某些对控制精度要求更 2 高的场合。针对该控制精度问题，通过对模糊PI控制策略的隶属度函 数进行改进, 提升控制精度。通过MATLAB/SIMULINK仿真实验进行验证, 论证其良好的补偿性能, 更好的满足电力系统 对无功功率补偿的要求。
\end{abstract}

关键词: 改进型模糊PI控制, 静止无功发生器, 隶属度函数, 无功功率补偿

\section{1. 引言}

对于无功功率补偿设备来说, 静止无功发生器有着不 可或缺的作用。静止无功发生器是通过产生或者消耗无功 功率, 进而维持电网水平稳定。由于静止无功发生器具有 良好的性能, 其是FACTS设备中重要的一部分。通过国际
专家和学者不断的努力, 此种设备的性能得到了很大的提 升, 控制精度得到明显的改善 [1]。

静止无功发生器的核心在于其控制策略上, 其控制策 略经过长时间的研究在不断的演变着。文献 [2] 介绍了PID 控制策略下STATCOM的工作原理以及实现无功补偿的过程。 PID的应用范围广、参数易整定。但其自身缺点比多, 无 法进行连续的自身整定模式，对非线性、时变、耦合以及 
参数和结构不确定的复杂过程, 工作效果不理想。美国的 L. A. Zadeh创建的模糊数学理论在 20 世纪 $60 、 70$ 年代得到 了大量的应用, 此方法也在STATCOM中进行了应用。模糊 控制理论模仿人工智能的方式调节无功功率的大小, 文献 [3] 对相关内容作了介绍。但由于模糊控制缺乏系统性, 无法保证解决模糊控制中稳定性和鲁棒性问题。模糊PI 控制是一种较为理想的控制策略, 其结合了模糊控制理论 和PI控制策略, 吸收两者优点。刚好的解决了其稳定性和 鲁棒性问题。为了扩大使用范围, 对模糊PI控制策略进行 优化, 通过调整隶属度函数优化控制效果。常规的隶属度 函数是均匀分布的, 本文采用的隶属度函数是不均与的, 根据实际的经验, 适当的调整区间范围, 使调整输入无功 功率的大小更趋于实际大小, 补偿的效果更为明显, 补偿 性能得到改善, 基本控制策略依旧是模糊PI控制策略, 对 此策略进行优化。

本文的主要工作是详细论述了 STATCOM在 $\mathrm{d}-\mathrm{q}$ 坐标系 下的数学模型, 通过调整隶属度函数的区间范围, 对补偿 效果进行优化, 与常规的模糊PI控制器进行对比, 通过 MATLAB/Simulink进行仿真验证。证明改进型模糊PI控制 方式的合理性和有效性。

\section{2. $d-q$ 坐标系下STATCOM的数学模型}

对STATCOM进行等效, 其等效电路图见图1, 根据输入 量和输出量来建立 $S V G$ 的相关数学模型 [4], 假设电阻 $R$ 为 在STATCOM中桥式变流器导通电阻以及相关的各种线路电 阻的总和, $X$ 为电网系统所连接的电抗器, 根据电压建立 STATCOM方程。

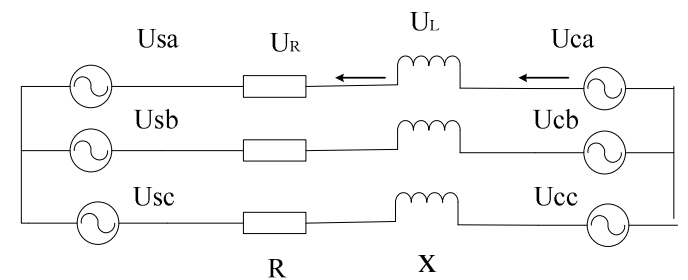

图1 STATCOM的三相等效电路图。

$\mathrm{u}_{\mathrm{sa}}, \mathrm{u}_{\mathrm{sb}}, \mathrm{u}_{\mathrm{sc}}$ 分别假设为系统的三相电压, 且其是对称的, 即电网电压的瞬时值见公式1:

$$
\begin{aligned}
& \left\{\begin{array}{l}
\mathrm{u}_{\mathrm{sa}}(\mathrm{t})=U_{s} \sin (\omega t) \\
\mathrm{uss}_{\mathrm{s}}(\mathrm{t})=U_{s} \sin (\omega t-2 \pi / 3) \\
\mathrm{u}_{\mathrm{sc}}(\mathrm{t})=U_{s} \sin (\omega t+2 \pi / 3)
\end{array}\right\} \\
& \left\{\begin{aligned}
\mathrm{u}_{\mathrm{ca}}(\mathrm{t}) & =K u_{d c} \sin (w t-\delta) \\
& =\sqrt{2} U c \sin (w t-\delta) \\
\mathrm{u}_{\mathrm{cb}}(\mathrm{t}) & =K u_{d c} \sin (w t-2 \pi / 3-\delta) \\
& =\sqrt{2} U c \sin (w t-2 \pi / 3-\delta) \\
\mathrm{u}_{\mathrm{cc}}(\mathrm{t}) & =K u_{d c} \sin (w t+2 \pi / 3-\delta) \\
& =\sqrt{2} U c \sin (w t+2 \pi / 3-\delta)
\end{aligned}\right\}
\end{aligned}
$$

根据基尔霍夫电压和电流就可以列出STATCOM三相的 回路方程。

$$
\left\{\begin{array}{l}
L \frac{\mathrm{d} i_{a}(t)}{d t}=u_{c a}(t)-u_{s a}(t)-R i_{a}(t) \\
L \frac{\mathrm{d} i_{b}(t)}{d t}=u_{c b}(t)-u_{s b}(t)-R i_{b}(t) \\
L \frac{\mathrm{d} i_{c}(t)}{d t}=u_{c c}(t)-u_{s c}(t)-R i_{c}(t)
\end{array}\right\}
$$

对其进行 $d-q$ 变换, 就可以计算出旋转坐标系下, STATCOM的电压以及电流方程。

$$
\begin{gathered}
L \frac{\mathrm{d} i_{\mathrm{d}}(t)}{d t}=u_{c d}(t)-R i_{d}(t)+\omega L i_{q}(t) \\
L \frac{\mathrm{d} i_{q}(t)}{d t}=u_{c q}(t)-\omega L i_{d}(t)-R i_{q}(t)+\sqrt{3} U_{s}
\end{gathered}
$$

式中

$$
T=\sqrt{\frac{2}{3}}\left[\begin{array}{lll}
\cos \omega t & \cos (\omega t-2 \pi / 3) & \cos (\omega t+2 \pi / 3) \\
\sin \omega t & \sin (\omega t-2 \pi / 3) & \sin (\omega t+2 \pi / 3)
\end{array}\right]
$$

对相关式子进行一系列的化简, 得到其帕克变换。

$$
\mathrm{T}=\sqrt{\frac{2}{3}}\left[\begin{array}{cc}
\cos \omega t & \sin \omega t \\
\cos (\omega t-2 \pi / 3) & \sin (\omega t-2 \pi / 3) \\
\cos (\omega t+2 \pi / 3) & \sin (\omega t+2 \pi / 3)
\end{array}\right]^{\mathrm{T}}
$$

式中 $w$ 一电网电压角频率。

对式4分析可以知道, 在 $\mathrm{d}-\mathrm{q}$ 坐标系下, 电流之间并不 是独立存在的, 其存在着交叉耦合。STATCOM系统是一个 耦合系统。在设计STATCOM的时候, 根据STATCOM交/直流 侧关于瞬时功率平衡的原理, 推到直流侧的电容电压, 其 方程为:

$$
\frac{\mathrm{d} u_{d c}}{d t}=\frac{3}{2} \frac{u d i_{d}+u_{q} i_{q}-\left(i_{d}^{2}+i_{q}^{2}\right) R}{C u_{d c}}-\frac{u_{d c}}{C R_{d c}}
$$

式中 $\mathrm{R}_{\mathrm{dc}}$ 一直流侧电容等效损耗电阻 $\mathrm{u}_{\mathrm{dc}}$ 一直流侧电容电压

式4和式 7 为STATCOM在旋转坐标系下的数学模型 [5]。 在旋转坐标系下, 建立起来的系统数学模型是一个两输入 两输出且其相互耦合的非线性系统。

\section{3. 模糊PI控制器的设计}

根据模糊PI控制器的原理, 画出其结构框图, 如图2 所示; 选取接入点的电压参考值, 与实际值的偏差作比较, 计算出变化趋势 $\mathrm{e}$; 还需知道接入点电压参考值和实际值 的偏差变化趋势 $\triangle \mathrm{e}$, 使用模糊推理的方法进行模糊运算, 
得出当前时刻的 $\triangle K_{\mathrm{i}}$ 和 $\triangle K_{\mathrm{p}}$, 进而来实现PI参数的最优调 整 [6]。即

$$
\begin{gathered}
K_{P}=K_{p}^{\prime}+\Delta K_{P} \\
K_{i}=K_{i}^{\prime}+\Delta K_{i}
\end{gathered}
$$

式中 $K^{\prime}{ }_{p}, K^{\prime}{ }_{i}$ 为控制器整定值, 采用Ziegler-Nichols 对预整定值进行求解。

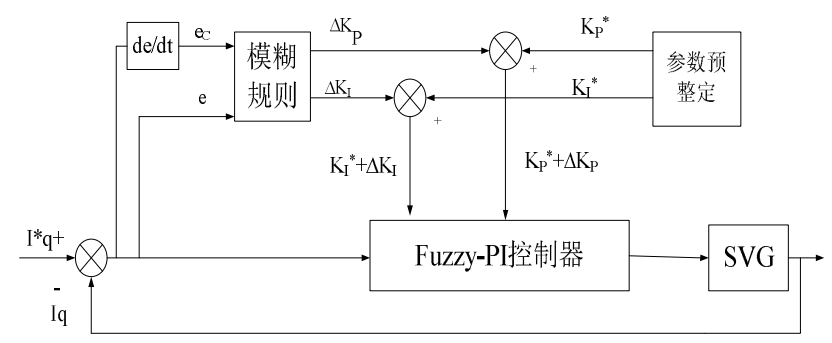

图2 模糊PI控制结构框图。

进行Fuzzy-PI控制器的设计, , 先置 $K{ }_{p}=K{ }{ }_{i}=0$, 再增 加 $K^{\prime}$, 直到系统发生震荡, 在此临界状态得到的 $K^{\prime}$ 值就 是 $K_{\text {cr }}$, 其振荡周期是 $T_{\text {cr }}$ 。此时, 可确定控制器参数为 $\mathrm{K}^{\prime}{ }_{\mathrm{p}}=0.45 \mathrm{~K}_{\mathrm{cr}}, \mathrm{K}^{\prime}{ }_{\mathrm{i}}=0.83 \mathrm{~T}_{\mathrm{cr}}$ 。在确定 $\mathrm{K}^{\prime}{ }_{\mathrm{p}}$ 和 $\mathrm{K}^{\prime}{ }_{\mathrm{i}}$ 值后, 就可以开 始设计模糊PI控制器, 相关设计内容主要由三个部分组成。

\section{1. 模糊化}

模糊控制量的输入变量以及输出变量都必须是精确 量, 然而模糊推理针对的是模糊量, 所以必须模糊化处理 相应的输入量。将输入变量和输出变量的语言值均分为相 应的7个语言值: $\{\mathrm{NB}, \mathrm{NM}, \mathrm{NS}, 0, \mathrm{PS}, \mathrm{PM}, \mathrm{PB}\}$, 隶属度 函数采用灵敏性强的三角函数 [7], 如图3所示, 各变量的 模糊量分别为 $E, E C$, 的 $\triangle K_{\mathrm{i}} 、 \triangle K_{\mathrm{p}}$, 其论域均为 $[-4,-3$, $-2,-1,0,1,2,3,4]$ 。

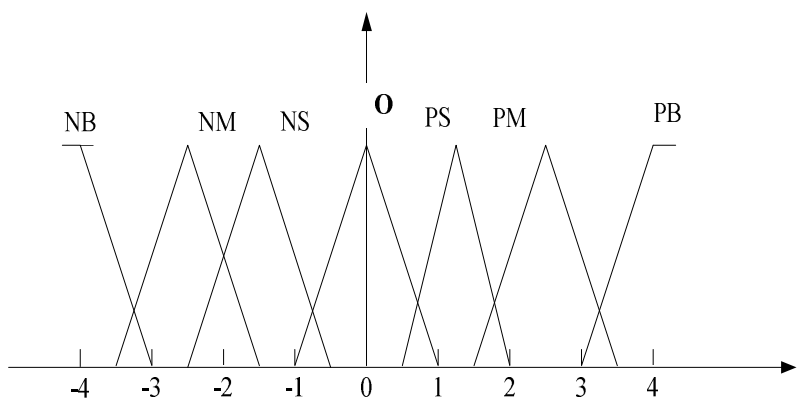

图3 隶属度函数。

\section{2. 参数整定规则的确定及模糊推理}

模糊推理的核心问题就是如何设置控制器参数的整 定规则, 其是由长期的现场调试经验以及相关领域专家的 经验所得。当系统出现偏差之后, 此时控制器发挥其控制 作用, 以防止偏差增大。根据实际对象的特征以及长期丰 富的调试经验, 进而总结出关于 $\triangle K_{i} 、 \triangle K_{\mathrm{p}}$ 两个参数的模 糊控制规则，如表1、表2所示。

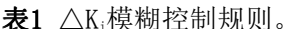

\begin{tabular}{lllllllll}
\hline \multirow{2}{*}{$\mathrm{U}$} & & $\mathbf{e}_{\mathbf{c}}$ & & & & & \\
\cline { 3 - 8 } & & NB & NM & NS & 0 & PS & PM & PB \\
\hline \multirow{4}{*}{} & NB & PB & PB & NB & PM & PS & PS & 0 \\
& NM & PB & PB & NM & PM & PS & 0 & 0 \\
& NS & PM & PM & NS & PS & 0 & NS & NM \\
& 0 & PM & PS & 0 & 0 & NS & NM & NM \\
& PS & PS & PS & 0 & NS & NS & NM & NM \\
& PM & 0 & 0 & NS & NM & NM & NM & NB \\
& PB & 0 & NS & NS & NM & NM & NB & NB \\
\hline
\end{tabular}

表2 $\triangle K_{\mathrm{p}}$ 模糊控制规则。

\begin{tabular}{lllllllll}
\hline \multirow{2}{*}{$\mathrm{U}$} & & $\mathbf{e}_{\mathrm{c}}$ & & & & & & \\
\cline { 2 - 8 } & & NB & NM & NS & 0 & PS & PM & PB \\
\hline \multirow{4}{*}{} & NB & PB & PB & NB & PM & PS & PS & 0 \\
& NM & PB & PB & NM & PM & PS & 0 & 0 \\
& NS & PM & PM & NS & PS & 0 & NS & NM \\
& 0 & PM & PS & 0 & 0 & NS & NM & NM \\
& PS & PS & PS & 0 & NS & NS & NM & NM \\
& PM & 0 & 0 & NS & NM & NM & NM & NB \\
& PB & 0 & NS & NS & NM & NM & NB & NB \\
\hline
\end{tabular}

对输入的 $\triangle \mathrm{e}$ 和e两个参数, 选取相应的语言值后, 按照整定规则表, 通 过采用公式法进行模糊决策, 可以分别得出 $\Delta K_{\mathrm{i}} 、 \Delta K_{\mathrm{p}}$ 两个修正参数的 模糊量。

\section{3. 去模糊化}

对模糊推理完成以后, 根据Fuzzy-PI控制器整定出两 个修正参数以后, 需要对其进行去模糊化处理, 此时得到 的是精确量, 进一步计算输出的控制量。去模糊化处理主 要采用最大隶属度法和重心法 [8]。本文控制器所采用的 是重心法, 求取输出量的精确值。

$$
\begin{gathered}
\Delta \mathrm{K}_{\mathrm{P}}=f_{P}(e, \Delta e)=\frac{\sum_{j=1}^{n} \mu_{j}(e, \Delta e) \Delta K_{P j}}{\sum_{j=1}^{n} \mu_{j}(e, \Delta e)} \\
\Delta \mathrm{K}_{\mathrm{I}}=f_{I}(e, \Delta e)=\frac{\sum_{j=1}^{n} \mu_{j}(e, \Delta e) \Delta K_{I j}}{\sum_{j=1}^{n} \mu_{j}(e, \Delta e)}
\end{gathered}
$$

根据 $K_{\mathrm{P}}=K_{\mathrm{P}}{ }^{\prime}+\triangle K_{\mathrm{p}}, K_{\mathrm{I}}=\mathrm{K}_{\mathrm{I}}{ }^{\prime}+\triangle K_{\mathrm{i}}$ 可得 $K_{\mathrm{P}} \mathrm{K}_{\mathrm{I}}$

$$
\begin{gathered}
\Delta \mathrm{K}_{\mathrm{P}}=K_{p}^{*}+\frac{\sum_{j=1}^{n} \mu_{j}(e, \Delta e) \Delta K_{P j}}{\sum_{j=1}^{n} \mu_{j}(e, \Delta e)} \\
\Delta \mathrm{K}_{\mathrm{I}}=K_{I}^{*}+\frac{\sum_{j=1}^{n} \mu_{j}(e, \Delta e) \Delta K_{I j}}{\sum_{j=1}^{n} \mu_{j}(e, \Delta e)}
\end{gathered}
$$


当系统在线运行的时候, 控制系统根据模糊逻辑规则 计算出的结果来进行处理、查表以及完成运算, 实现参数 的在线自整定。

\section{4. 对隶属度函数进行改进}

静止无功发生器的核心在于其控制策略上, 对于模糊 PI控制策略的STATCOM而言, 主要对模糊控制这一部分进 行优化, 本文主要对专家定义语言变量的隶属度函数进行 改进。隶属度函数的确定过程既有主观性又有客观性。隶 属度函数的实质反应的是函数的渐变性。设计常规的隶属
度函数时, 将每个语言变量进行均与的分布。此种方式的 误差比较大, 为了提高系统的控制精度, 经过相关学者的 大量研究, 将语言变量进行不均匀的分布更加符合实际情 况。离参照点越近的时候, 隶属度函数曲线越陡。即离参 照点越近越敏感, 当系统在 0 值附近时, 需要控制策略更 加敏感, 补偿效果更好, 因此让其更陡些。相比于常规模 糊控制器的隶属度函数, 本文做了相应的改进, 如图4所 示。将此隶属度函数应用在 STATCOM 中, 并在 MATLAB/Simulink中进行仿真验证 [9]。

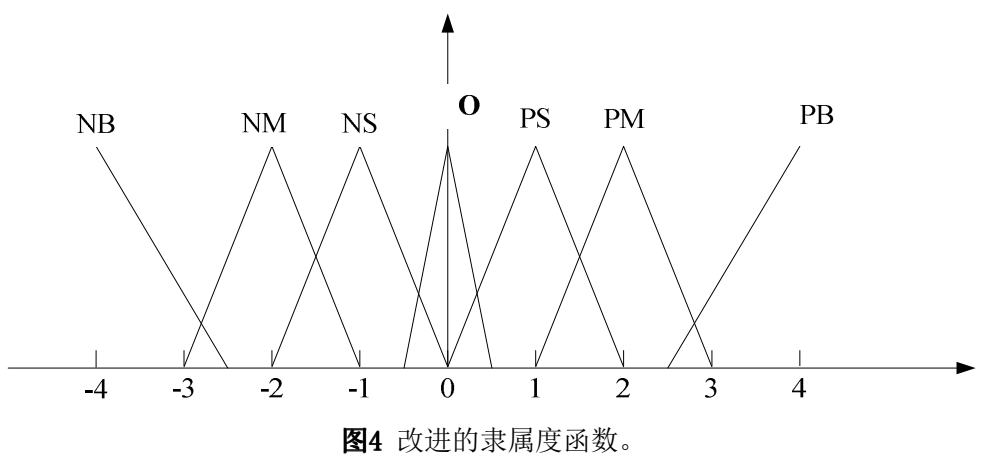

\section{5. 仿真与验证}

对改进型模糊PI控制算法的补偿效果进行论证，检验 其有效性和可行性。还需要对模糊PI控制策略和改进型模 糊PI控制策略两种控制策略的仿真进行对比。系统仿真的 设计参数如下: 系统电压 $500 \mathrm{~V}$; 频率 $50 \mathrm{~Hz}$; 直流侧电容 1500 $\mu \mathrm{F}$; SPWM载波频率: $5 \mathrm{KHz}$; 耦合变压器和滤波电感等效 电感为: $5 \mathrm{mH}$ 、等效电阻 $0.3 \Omega$; 公共连接点电压控制器的
初始参数: $K p=3, K_{I}=150$; 直流侧电容电压控制器的初始 参数: $\mathrm{Kp}=1, \mathrm{~K}_{\mathrm{I}}=100$ 。

为了检验控制器的动态性能和自适应性能, 在 STATCOM和系统电网的公共连接点模拟电压跌落的情况, 改变STATCOM系统中的电气参数来研究系统工作状态的变 化 [10]。如图5所示, 在 $\mathrm{t}=0.15 \mathrm{~s}$ 时给系统添加无功负荷, 可以看出两种控制策略下对系统负荷变化的响应曲线 (标 幺值）； $\mathrm{t}=0.3 \mathrm{~s}$ 时，系统基本恢复。

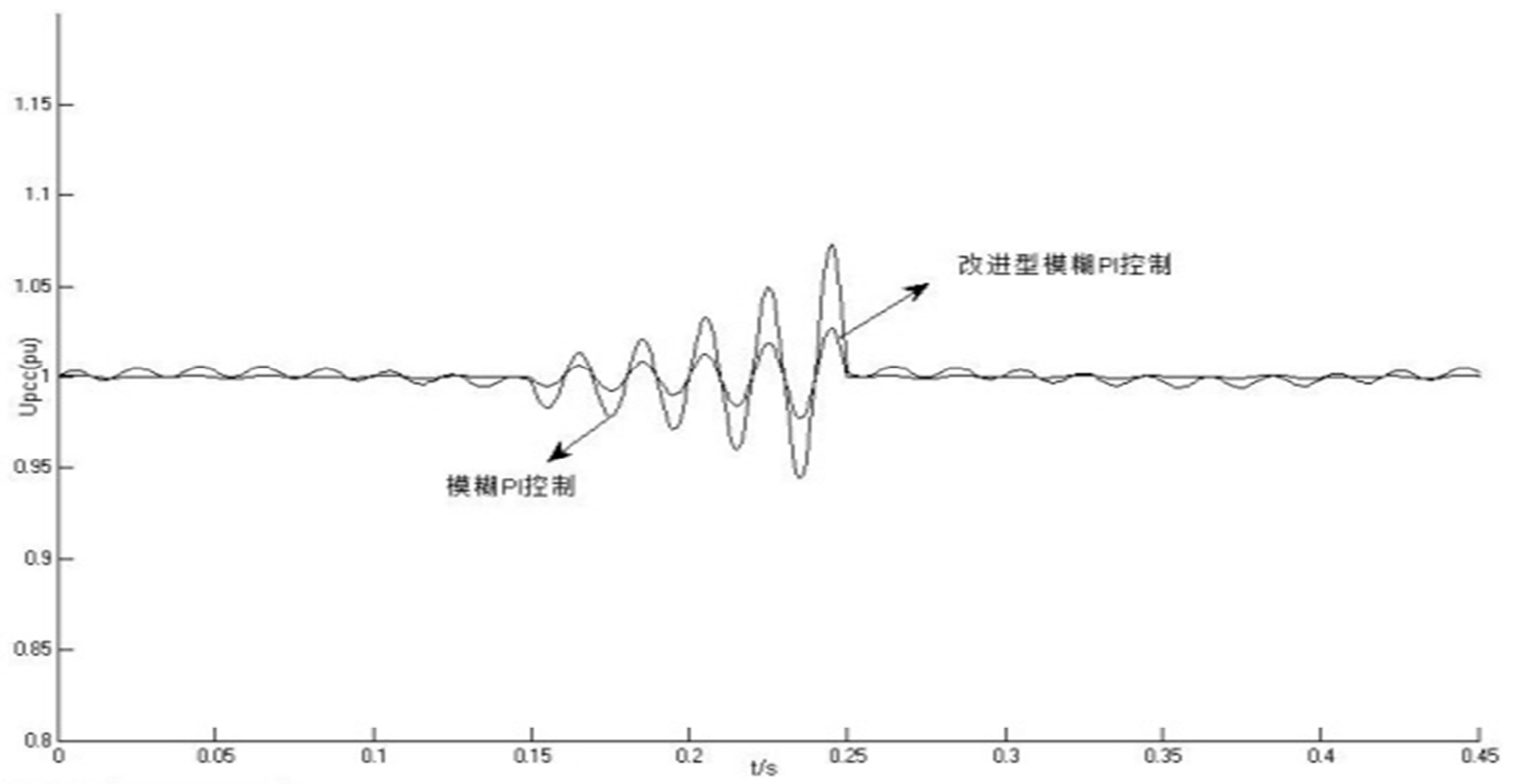

图5 公共连接点电压响应曲线。 
分析图5可以发现, 改进型模糊PI控制动态性能更好, 其超调量小而且调节时间也比较短。

从图6 是有关直流侧电容电压的波形图, 当有外界无 功负荷加到系统中时, 公共连接点的电压会下降, 此时 STATCOM需要输送无功电流, 进而使系统恢复到原来的电
压水平，保持系统恒定 [11]。对比两种控制策略，也就是 模糊PI控制和改进型模糊PI控制策略, 采用改进型模糊PI 控制这种策略时, 其直流侧电容电压的波形波动幅度不大, 具有良好的性能。

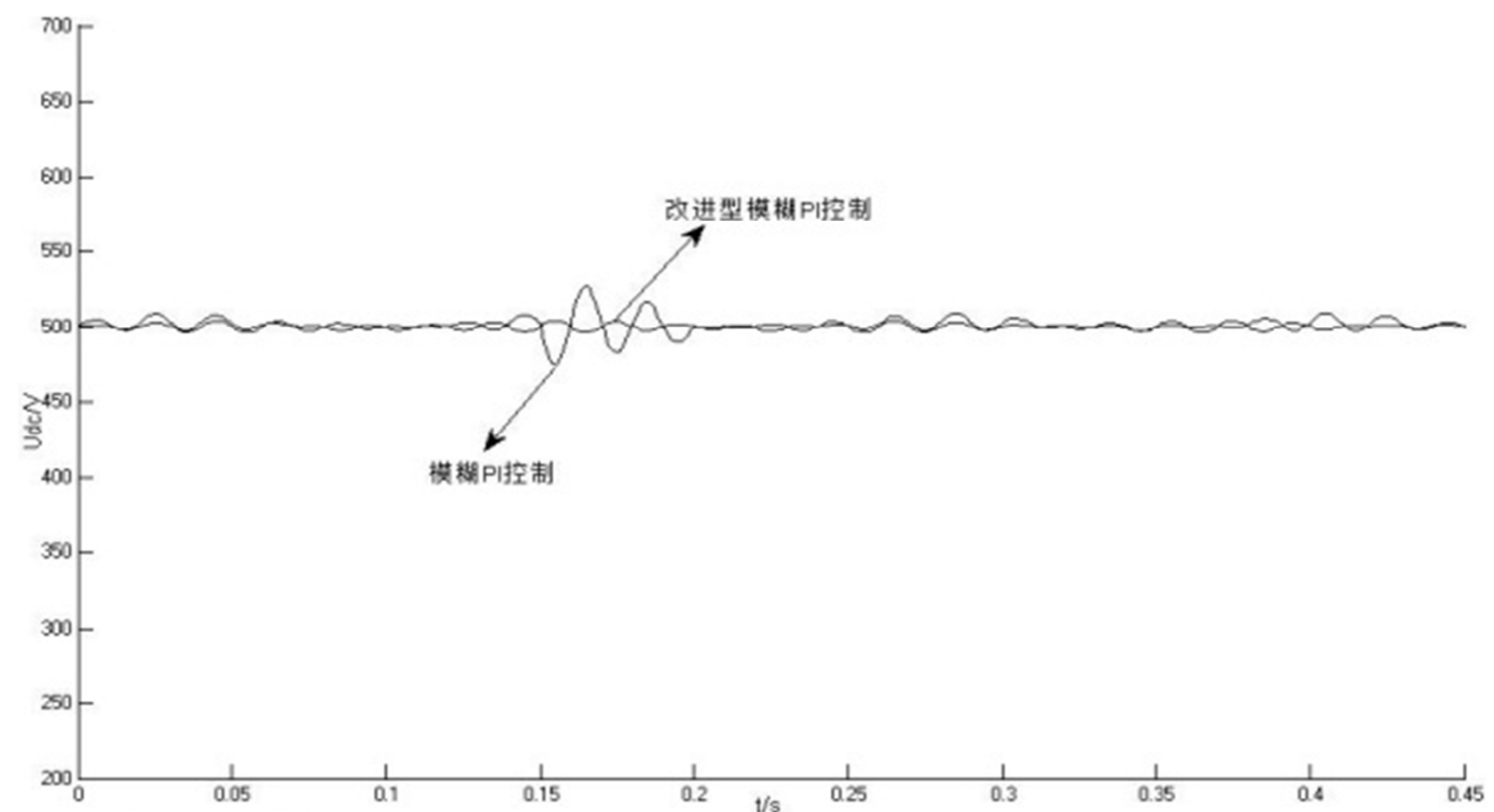

图6 直流侧电容电压波形。

为了更好的说明改进型模糊PI控制策略比模糊PI控制策略的动态性能和可行性更好, 改变系统参数, 将耦合变压 器和滤波电感等效电感参数设置为 $8 \mathrm{mH}$, 等效电阻设置为 $0.7 \Omega$ 。图 7 、图8分别给出了两种控制策略下, 公共连接点电 压以及直流侧电容电压波形。

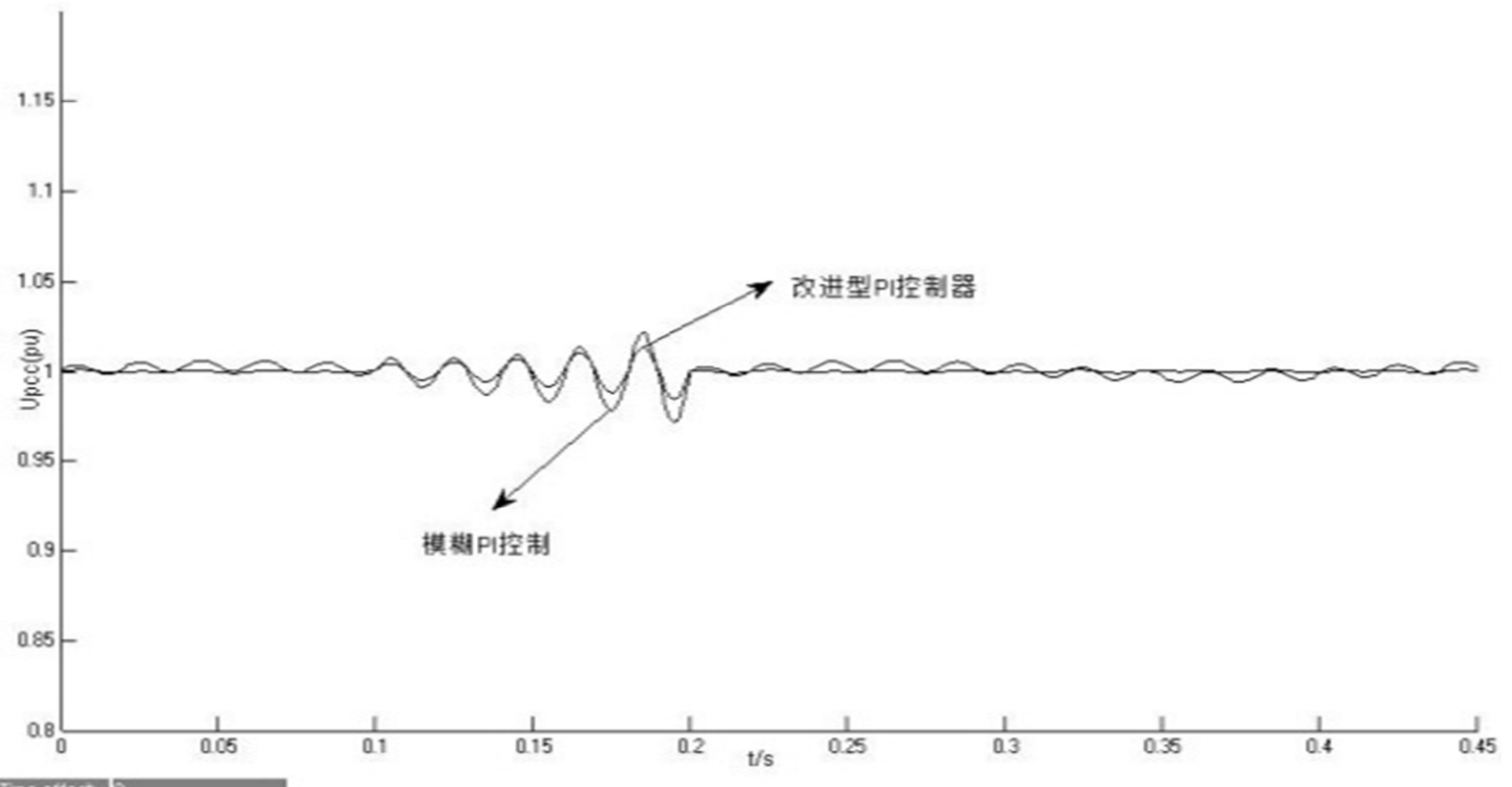

图7 公共连接点电压响应曲线。 


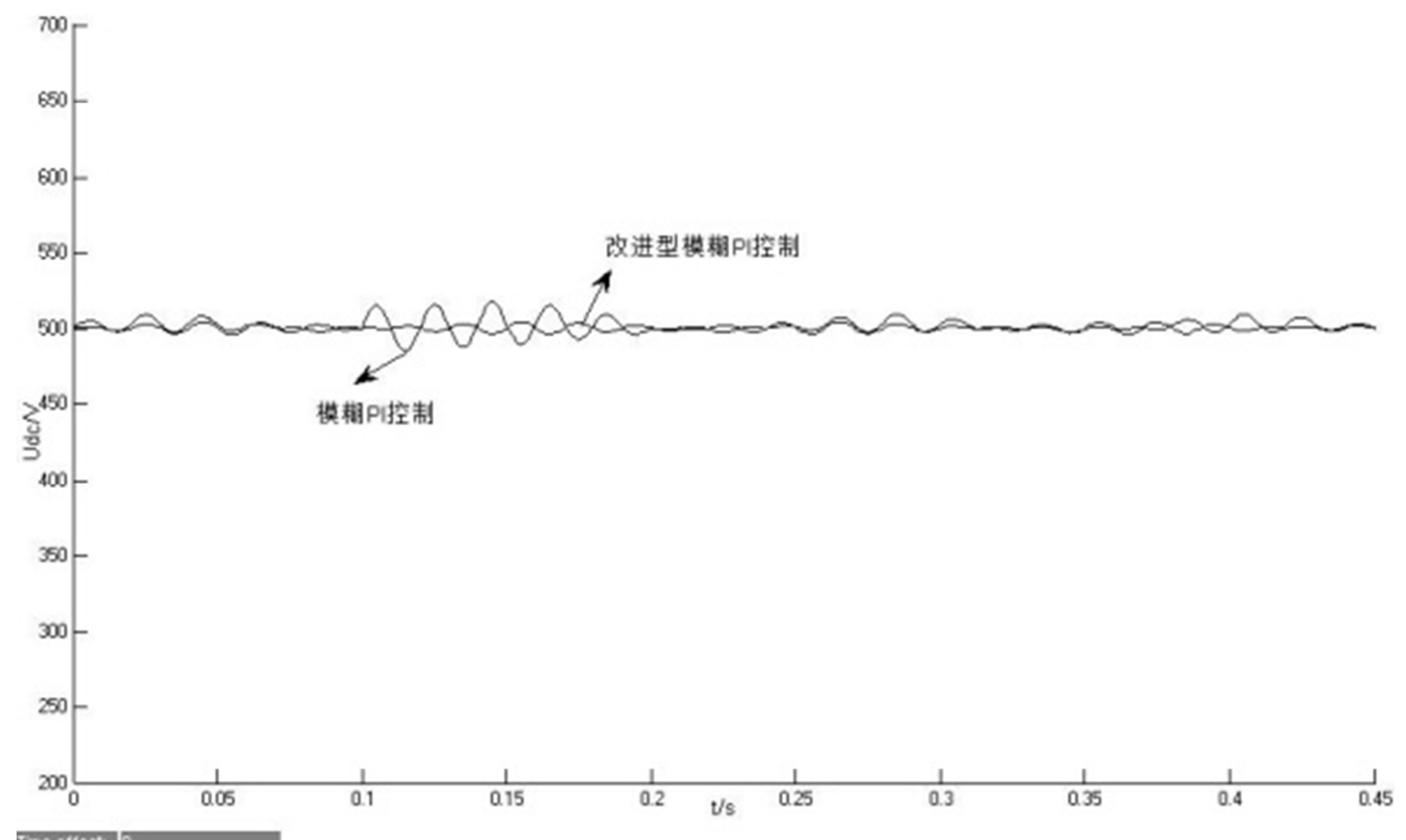

图8 直流侧电容电压波形。

从图7、图8可以看出, 系统等效参数发生变化时, 改 进型模糊PI控制策略可以使系统更好的恢复到原来的水 平, 没有受到等效系统参数的影响而影响, 其鲁棒性好, 补偿效果更好。

\section{6. 结论}

本文对模糊PI控制策略的隶属度函数进行了改进, 使 系统控制精度得到了提升, 在发挥模糊控制的优势的同时, 也对模糊控制在调节变化过程中出现的偏差以及偏差变 化率进行了最佳整定, 达到系统所需补偿的要求。模糊PI 控制可以消除静差, 对其隶属度函数进行改进, 使模糊PI 控制策略性能更加优良。满足控制精度要求更高的场合, 为STATCOM的应用提供了更为广阔的应用场合。

\section{参考文献}

[1] 罗承廉, 季勇, 刘遵义. 静止同步补偿器 (STATCOM) 的原理 与实现. 北京: 中国电力出版社, 2005。

[2] Hanzelka Z, Szpyra W, Cziker A, etal. Reactive power compensation[J].Electrical energy: technologies and application, 2012:371-398.

[3] A. Ghafour; M. R. Zolghadri; M. Ehsan; 0. Elmatboly; A. Homaifar Power Symposium. Fuzzy Controlled STATCOM for Improving the Power System Transient Stability [J]. Power Symposium, 2007:212-216.

[4] B. Singh; K. V. Srinivas. Fuzzy logic control with constant dc link voltage of $48-$ pulse VSC based STATCOM [J]. Power Eleconics, 2011: 1-7.

[5] 王兆安, 杨君, 刘进军. 谐波抑制与无功补偿 [M]. 北京: 机 械工业出版社，2004。

[6] S. Raman; R. Gokaraju; A. Jain. An adaptive fuzzy mho relay for phase backup protection with infeed from STATCOM $[J]$. Power and Energy Society General Meeting, 2013: 1-1.

[7] 邓明锋, 邓素英. 参数自整定模糊PI控制的级联STATCOM外 环电压控制 $[J]$. 电工技术. 2015（4）：100-103。

[8] M. Janaki; R. Thirumalaivasan; N. Prabhu. Energy function based Fuzzy Logic Discrete control with STATCOM for stability improvement $[\mathrm{J}]$. Advances in Electrical Engineering, 2014: 1-5.

[9] 储月娥, 吕宗伟. 模糊控制器的设计原理及其应用 $[\mathrm{J}]$. 自动 化与仪表. 2005（5）：42-48。

[10］常鲜戎, 陈新超, 季国庆, 邓燕山. DSTATCOM直流侧电压简 易模糊PI控制研究 $[J]$. 电能计量与负荷控制. 2015（14）: 11-15。

[11] 曾光, 柯敏倩, 张静刚. 用于静止无功补偿器的模糊-PID 控制方法研究 $[J]$. 电力电子技术，2006, 39（5）：89-95。 